\title{
Orientation Diffusions
}

\author{
Pietro Perona
}

\begin{abstract}
Diffusions are useful for image processing and computer vision because they provide a convenient way of smoothing noisy data, analyzing images at multiple scales, and enhancing discontinuities. A number of diffusions of image brightness have been defined and studied so far; they may be applied to scalar and vector-valued quantities that are naturally associated with intervals of either the real line, or other flat manifolds. Some quantities of interest in computer vision, and other areas of engineering that deal with images, are defined on curved manifolds; typical examples are orientation and hue that are defined on the circle. Generalizing brightness diffusions to orientation is not straightforward, especially in the case where a discrete implementation is sought. An example of what may go wrong is presented.

A method is proposed to define diffusions of orientation-like quantities. First a definition in the continuum is discussed, then a discrete orientation diffusion is proposed. The behavior of such diffusions is explored both analytically and experimentally. It is shown how such orientation diffusions contain a nonlinearity that is reminiscent of edge-process and anisotropic diffusion. A number of open questions are proposed at the end.
\end{abstract}

Index Terms-Orientation analysis, texture analysis, diffusions, scale-space.

\section{INTRODUCTION}

\section{A. Why Orientation Diffusions?}

C ONSIDER the image in Fig. 1. It is quite clear that over most of the area of this picture the important information is contained in the orientation of the lines, rather than in the brightness values.

It is not easy, sometimes not possible, to determine the local orientation of the image using local operators. In Fig. 2 the orientations detected using a simple gradient-based method are shown; such an orientation image is quite noisy. In order to obtain a more precise determination of orientation one may use more sophisticated filtering schemes, for example one may use Gabor-like filters that are tuned to the specific frequency that is present in the image (see, e.g., [1]), however, by doing so one gives up locality (such filters are larger), generality (such filters need to be tuned for a specific frequency), and one may not be able to detect interesting events such as the two orientation singularities that are present in the image. It would be nice to be able to detect a noisy signal using very local operators, and then "average" this information locally

Manuscript received November 1, 1996; revised March 21, 1997. The associate editor coordinating the review of this manuscript and approving it for publication was Dr. Guillermo Sapiro.

The author is with the California Institute of Technology, Pasadena, CA 91125 USA, and with the University of Padova, Padova, Italy (e-mail: perona@vision.caltech.edu).

Publisher Item Identifier S 1057-7149(98)01780-1.
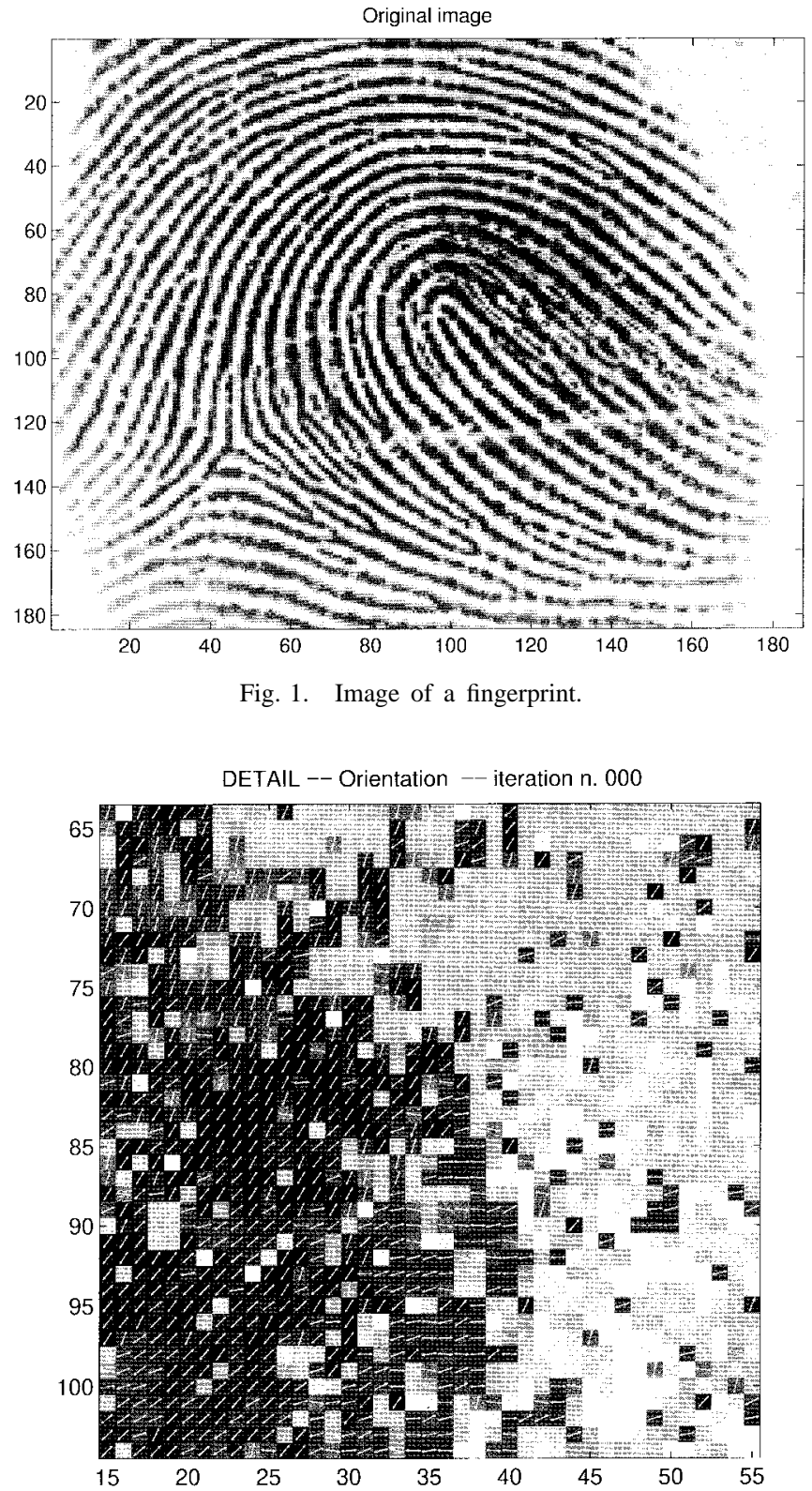

Fig. 2. Orientations detected on a detail of the image in Fig. 1 by using the gradient of a slightly blurred version of the image.

and adaptively in order to achieve both good noise rejection and localization of interesting singularities.

A similar problem has been faced for a long time by researchers working on brightness images. Lowpass filtering, or, equivalently, diffusing the image, has long been seen as a convenient way of rejecting noise and reconstructing the underlying data. By analogy, it may be a good idea to blur, or diffuse, orientation information in order to obtain more reliable 
information based on a neighborhood of each point.

Another reason for diffusing images is that diffusions naturally produce a "scale-space," i.e., a fine-to-coarse family of derived images. The main structures present in the image are easier to detect in the coarser scale portions of the scalespace, while the finer scale portions, closer to the original image, are more suitable for localization tasks [2]-[4] (for a comprehensive review of work on diffusions, see [5]). It is reasonable to try and define scale-space operators for orientation images as well.

A number of researchers [6]-[10] have proposed methods for diffusing, or lowpass filtering, orientation images. These methods may be, in essence, reduced to a simple three-step process. First, embed orientation in the plane via the map $\theta \rightarrow w=[\cos (\theta), \sin (\theta)]$; second, diffuse in the plane for a time $t$; third, project back to the circle via the map $w_{t}=[x, y] \rightarrow \arctan (y / x)$. There are two caveats: In the cited works the norm of the orientation vector $w$ is not restricted to be equal to one, rather it is set to represent image contrast; here we are purely interested in orientation, therefore we will use norm one vectors. Moreover, some of the authors referenced above diffuse an "orientation tensor," rather than the orientation vector $w$; as pointed out in $[9, \mathrm{p}$. 229], the two are equivalent if the quantity being diffused is an orientation defined in the plane. As it will be shown in Section II, this process has two effects that may be unwanted: It is not consistent if it is iterated, and it produces large-scale changes in the topology of the orientation image with large jumps in the value of orientation.

\section{B. Representing Orientation}

Before trying to define orientation diffusions we need to clarify the concept of orientation. Orientations may be associated with points $\theta$ on a circle; however, one has to be aware of the fact that there are two main "types" of orientation. After being rotated by $180^{\circ}$, a brightness step edge looks different; however, a line will look the same. The step edge is $360^{\circ}$ periodic, while the line is $180^{\circ}$ periodic. Hue is $2 \pi$-periodic as well. There may be quantities that are $\pi / 2$-periodic, for example cross-junctions as depicted in Fig. 3.

Granlund and Knuttson [9] have proposed to embed each one of these periodic quantities in the $(-\pi, \pi)$ circle by appropriate scaling of the orientation variable. For example, the map $\theta \rightarrow 2 \theta$ maps line orientation onto the circle, as shown in Fig. 3 (center). Analogous embedding may be done on the complex plane as $e^{i a \theta}$ by appropriate choice of the constant $a$. As a result we may think of any orientation in a unified way as a quantity that is defined on the circle. In the rest of this paper we will therefore not need to worry any longer about the problem of which orientation we are using and we will use $\theta \in[-\pi, \pi]$ as our variable representing orientation.

\section{CONTINUOUS SPACE}

The most straightforward idea for extending brightness diffusions to an orientation image $\theta_{0}(x, y)$ is to ignore the fact that $\theta$ is defined on the circle, rather than an interval of the



Fig. 3. There are different kinds of oriented structures in images. Some are $2 \pi$-periodic, some are $\pi$-periodic, others may have even smaller periods. This dishomogeneity is more apparent than real; as Granlund, Knuttson, and collaborators have suggested, whatever its length the period may be mapped onto the circle thus obtaining a unified representation.

line, and proceed as in the conventional linear diffusion case

$$
\begin{aligned}
\bar{\theta}(x, y, 0) & =\theta_{0}(x, y) \\
\bar{\theta}_{t}(x, y, t) & =\triangle \bar{\theta}(x, y, t) .
\end{aligned}
$$

Is this a good idea? In this section it will be shown that this is indeed a good method in the continuous case, and that it is related to the traditional embed-diffuse-project method that was sketched in the introduction. At the end of the section it will be shown, however, that a naive discretization of this method does not work; discrete orientation diffusions will be introduced in Section III.

As discussed in the introduction, a popular way of diffusing orientation is to diffuse the components of an orientation vector (equivalently, an orientation tensor) that is embedded in a Euclidean space.

Definition 1: Call $\mathcal{D}_{[t]}^{\prime}$ the operator that maps the function $\theta_{0}(x, y)$ into the function $\theta(x, y, t)$ by the sequence of embedding, diffusion and projection

$$
\begin{aligned}
w(x, y, 0) & =e^{i \theta_{0}(x, y) \quad \text { embedding }} \\
w_{t}(x, y, t) & =\lambda \triangle w(x, y, t) \quad \text { diffusion } \\
\theta(x, y, t) & =\text { phase } w(x, y, t) \quad \text { projection }
\end{aligned}
$$

where $w_{t}$ indicates $\partial w / \partial t$ (this notation will be used throughout the paper), and $\lambda$ is a positive constant determining the velocity of the diffusion.

Observation 1: This technique does not have the semigroup property, i.e., it is not necessarily true that when $0<t_{1}<t_{2}$ we have $\theta\left(x, y, t_{2}\right)=\mathcal{D}_{\left[t_{2}-t_{1}\right]}^{\prime} \theta\left(x, y, t_{1}\right)$. See the example in Fig. 4. If one wants a consistent behavior one has to use both phase and magnitude of $w\left(x, y, t_{1}\right)$ as the initial condition of further diffusion.

Observation 2: This technique may produce results that are "topologically wrong." In Fig. 4, fourth and fifth columns, it is demonstrated that it may "unwind" an orientation loop while smoothing it. In the process, around location 45 the orientation suddenly jumps by $\pi$.

It is possible, however, to define a related diffusion that preserves the semigroup property. The main problem with the traditional "plane-embedding" definition of orientation diffusions stems from the fact that the result of the diffusion in 

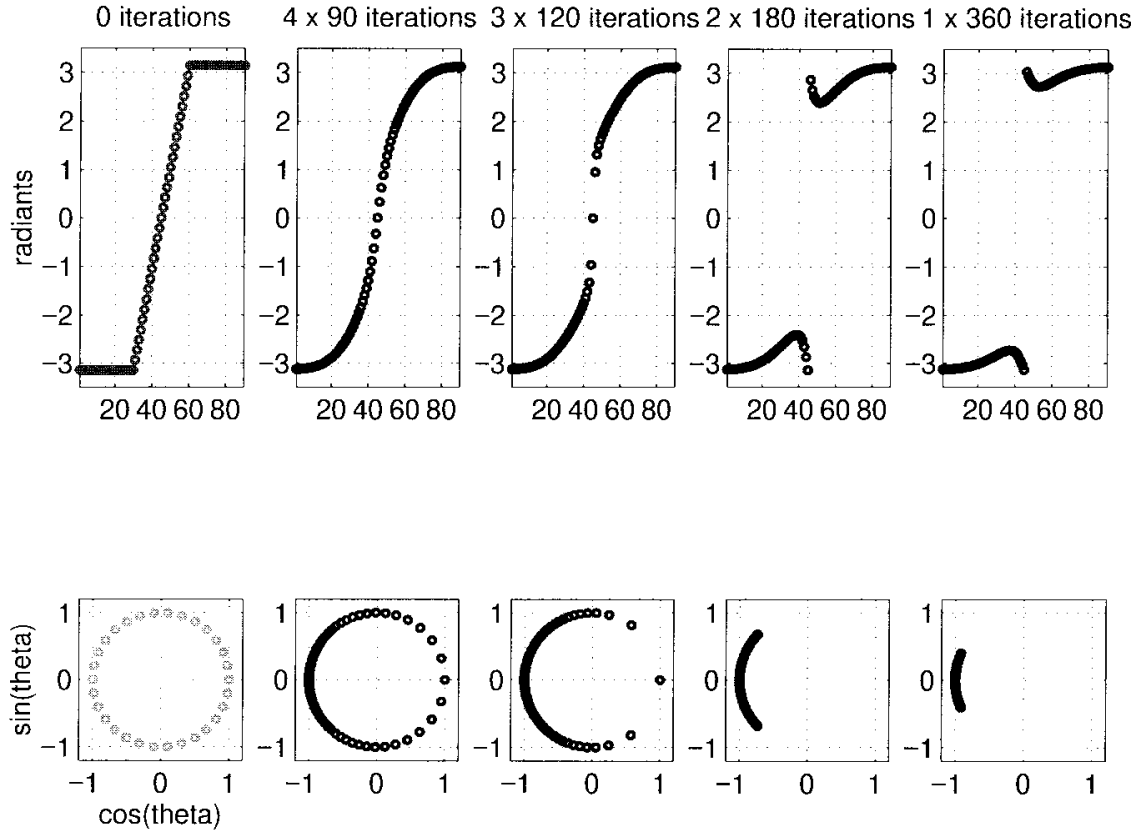

Fig. 4. Example of violation of the semigroup property. The diffusion is performed on the 1-D orientation signal represented in the far-left column (each signal is represented twice: as an angle [top row), and as a polar plot (bottom row)]. The signal is equal to $\pi$ (equivalently $-\pi$ ) at the boundaries, and undergoes a full rotation between the thirtieth and the sixtieth sample. The second to fifth columns show the result of applying $\mathcal{D}_{[90]}^{\prime}$ four times, $\mathcal{D}_{[120]}^{\prime}$ three times, etc., so that the total diffusion time is constant: $t=360$ time units. The last two columns present diffusion results that are qualitatively very different from the one in the second column. The third column shows a behavior that is close to critical. Notice that the result of diffusing the signal in the plane (the last column) is "wrong" because as a result of the diffusion the $2 \pi$ loop has disappeared, and the signal just oscillates around $\pi$. Compare with the result of applying $\mathcal{D} \prime_{[90]}^{4}$, where the kinks are smoothed while the topology of the loop is preserved.

the plane is projected back onto the circle only once, at the end of a time interval. As it may be noticed in Fig. 4 the result of repeating short diffusions and projection cycles is more "correct" than what is obtained via a single cycle composed of a long diffusion followed by a projection.

One might think of taking this last observation to the limit: alternate vanishingly short diffusion phases with projections onto the circle in order to obtain a consistent behavior.

Definition 2: Call $\mathcal{D}^{\prime k}$ the $k$-th power of $\mathcal{D}^{\prime}$, i.e., the operation of applying $\mathcal{D}^{\prime} k$ times. Then we may define $\mathcal{D}$ as the limit obtained applying $\mathcal{D}^{\prime}$ many times for very short intervals, as follows:

$$
\mathcal{D}_{[t]}=\lim _{\delta \rightarrow 0} \mathcal{D}_{[\delta]}^{\prime t / \delta}
$$

It turns out that this strategy generates a diffusion that has the semigroup property.

Proposition 1: The diffusion

$$
\theta(x, y, t)=\mathcal{D}_{[t]} \theta_{0}(x, y)
$$

enjoys the semigroup property.

Proof: We want to prove that $\mathcal{D}_{\left[t_{1}+t_{2}\right]}=\mathcal{D}_{\left[t_{2}\right]} \mathcal{D}_{\left[t_{1}\right]}$. Starting from the definition of $\mathcal{D}$ and dividing the limit into two limits one may verify this fact.

How do we calculate this diffusion in practice? It is possible, of course, to alternate short diffusion cycles with projections, as described above. There is a more natural implementation though. It is possible to prove that one may compute $\mathcal{D}_{[t]} \theta_{0}(x, y)$ by solving the usual heat diffusion equation:
Proposition 2: Call $\bar{\theta}(x, y, t)$ the solution of the diffusion equation

$$
\begin{aligned}
\bar{\theta}(x, y, 0) & =\theta_{0}(x, y) \\
\bar{\theta}_{t}(x, y, t) & =\triangle \bar{\theta}(x, y, t)
\end{aligned}
$$

and suppose that it is twice differentiable (the symbol $\triangle$ here indicates the "Laplacian" on the circle, one may calculate it by taking formally the spatial derivatives of $\theta$ ).

Then $\bar{\theta}(x, y, t)=\mathcal{D}_{[t]} \theta_{0}(x, y)$.

Proof: Let's start by taking a first-order Taylor expansion of $\mathcal{D}_{[\delta]}^{\prime} \theta(x, y, t)$ :

$$
\begin{aligned}
w(x, y, t) & =e^{i \theta(x, y, t)} \\
\theta(x, y, t+\delta) & =\text { phase } w(x, y, t+\delta) \\
& =\operatorname{Im} \log w(x, y, t+\delta) \\
w(x, y, t+\delta) & =w(x, y, t)+\delta \triangle w(x, y, t)+\mathcal{O}\left(\delta^{2}\right) .
\end{aligned}
$$

(We may take any determination of the complex log in the following calculations, provided that it is always the same).

We may compute aside the Laplacian of $w(x, y, t)$

$$
\begin{aligned}
\triangle w(x, y, t) & =w_{x x}+w_{y y} \\
& =\left(i \theta_{x x}-\theta_{x}^{2}+i \theta_{y y}-\theta_{y}^{2}\right) e^{i \theta} \\
& =\left(i \triangle \theta-|\nabla \theta|^{2}\right) e^{i \theta .}
\end{aligned}
$$


Let's now expand in Taylor series the complex log of $w(x, y, t+\delta)$ remembering that $\log (1+x)=1+x+\mathcal{O}\left(x^{2}\right)$.

$$
\begin{aligned}
\log w & (x, y, t+\delta) \\
& =\log \left(w(x, y, t)+\delta \triangle w(x, y, t)+\mathcal{O}\left(\delta^{2}\right)\right) \\
& =\log \left(e^{i \theta}\left[1+\delta\left(i \triangle \theta-|\nabla \theta|^{2}\right)+\mathcal{O}\left(\delta^{2}\right) e^{-i \theta}\right]\right) \\
& =i \theta+1+\delta\left(i \triangle \theta+|\nabla \theta|^{2}\right)+\mathcal{O}\left(\delta^{2}\right) e^{-i \theta}+\mathcal{O}\left(\delta^{2}\right) .
\end{aligned}
$$

Therefore

$$
\begin{aligned}
\theta(x, y, t+\delta) & =\operatorname{Im} \log w(x, y, t+\delta) \\
& =\theta(x, y, t)+\delta \triangle \theta(x, y, t)+\mathcal{O}\left(\delta^{2}\right) .
\end{aligned}
$$

From this and from the definition of $\mathcal{D}$

$$
\begin{aligned}
\mathcal{D}_{[\delta]} \theta & =\lim _{h \rightarrow 0} \mathcal{D}_{[h]}^{\prime \delta / h} \theta \\
& =\theta(x, y, t)+\lim _{h \rightarrow 0} \frac{\delta}{h} h \triangle \theta(x, y, t)+\mathcal{O}\left(\delta^{2}\right) \\
& =\theta(x, y, t)+\delta \triangle \theta(x, y, t)+\mathcal{O}\left(\delta^{2}\right)
\end{aligned}
$$

and therefore

$$
\theta_{t}=\lim _{\delta \rightarrow 0} \frac{\mathcal{D}_{[\delta]} \theta-\theta}{\delta}=\triangle \theta
$$

A caveat is that the derivatives in the Laplacian $\triangle$ should be taken keeping in mind that theta is periodic, and therefore that $0=2 \pi$.

In order to implement this diffusion on a computer one has to discretize it both in space and time. Unfortunately, is not clear how to discretize this diffusion on a lattice in $X$ and $Y$ : what is the discrete version of the Laplacian? Plain finite differences will not do for approximating the spatial derivatives of $\theta$.

One could think of using a plain finite-difference approximation of the Laplacian of (2) as is commonly done for brightness diffusions (for simplicity of notation a single space coordinate $x$ is used here):

$$
\begin{aligned}
\theta(x, t+1)= & \theta(x, t)+\lambda[\theta(x-1, t)-2 \theta(x, t)+\theta(x+1, t)] \\
= & \theta(x, t)+\lambda[\theta(x-1, t)-\theta(x, t)] \\
& +\lambda[\theta(x+1, t)-\theta(x, t)] .
\end{aligned}
$$

This equation is easy to interpret: the variable $\theta$ is incremented at the location $x$ by an amount that is proportional to the sum of the difference of $\theta(x)$ with its neighbors $\theta(x-1)$ and $\theta(x+1)$.

While this is a perfectly natural discretized algorithm when the quantity to be diffused is defined on a flat manifold, it leads to an ambiguity on the circle. Suppose that two adjacent lattice locations $A=x$ and $B=x+1$ have associated orientations $\theta_{A}=0$, and $\theta_{B}=\pi / 2$, say. The difference $\theta_{B}-\theta_{A}$ may be represented in two ways: $\pi / 2$ and $-3 \pi / 2$. For now this representation ambiguity is only apparent since both notations denote the same element of the circle. However, as soon as we multiply these quantities by a small scalar $\lambda$, in order to calculate the increment to be applied to $\theta$ as a consequence of the diffusion, we find ourselves in the embarrassing position of having to choose between a counterclockwise small increment, and a clockwise larger increment (see Fig. 5).
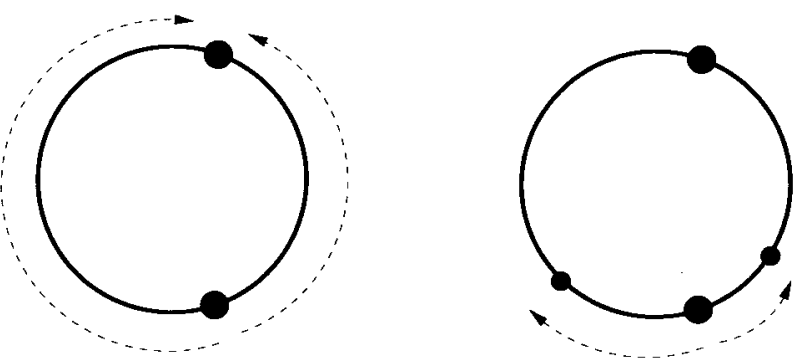

Fig. 5. The difference between two orientations may be expressed by two different notations, one positive and one negative, that denote, however, the same quantity. When such difference is multiplied by a small scalar we have an ambiguity.

Another problem is how to "bias" the diffusion to keep the solution sufficiently close to some constant "data" terms $\theta_{d}$. Also in this case finite differences, or distances, $d\left(\theta, \theta_{d}\right)$ have to be computed. This is another case where the differential definition of "distance" that is implicit in (2) is not helpful.

Also, is not clear how to define the diffusion in general. The hypothesis that the solution is twice differentiable makes the differential formulation useless in practice.

\section{DISCRETE SPACE}

In the previous section we have found a simple expression for the "natural" diffusion of orientation on a continuous spatial domain. We have seen that it is not possible to obtain the discrete version of this diffusion on a spatial lattice by simply taking spatial finite differences in order to approximate the spatial derivatives of $\theta$.

In order to solve this problem we need to take one step back and derive the notion of diffusion of orientation by revisiting the relationship between diffusions and variational problems.

First of all recall that the common diffusion equation $u_{t}=\Delta u$, where $u$ is a real-valued function defined on the plane (line), may be obtained as the "gradient descent" solution of the Euler equation of an energy, or cost, functional $\mathcal{E}(u)=\int_{x, y}|\nabla u|^{2} d x d y$.

The discrete analog (for the sake of convenience we may use one discrete spatial coordinate $k$ ) of such a cost function is $\mathcal{E}(u)=\frac{1}{2} \Sigma_{k}\left(u_{k+1}-u_{k}\right)^{2}$. The gradient descent strategy for minimizing such a cost function consists in the algorithm

$$
\frac{\partial}{\partial t} u_{k}=-\lambda \frac{\partial}{\partial u_{k}} \mathcal{E}(u)=\lambda\left(u_{k-1}-2 u_{k}+u_{k+1}\right)
$$

where $\lambda$ is a "velocity" parameter.

Observe that the quantity $u_{k-1}-2 u_{k}+u_{k+1}$ is a discretization of $\Delta u$, and $\lim _{\delta \rightarrow 0}\left(u_{x-\delta}-2 u_{x}+u_{x+\delta} / \delta^{2}\right)=\Delta u$. Moreover, the cost function or "energy" function is composed of the sum of pairwise terms $\left(u_{k+1}-u_{k}\right)^{2}$, i.e., it is the sum of the costs or energies associated to each pair of neighboring locations.

By analogy, we may be able to obtain a definition of a diffusion of orientation-like quantities on a discrete lattice if we manage to define the pairwise energy of two orientations. There are many reasonable definitions. If one uses the physical analogy with the energy associated to two superimposed magnets oriented as $\theta_{A}$ and $\theta_{B}$, then the pairwise energy is 
$\mathcal{E}\left(\theta_{A}, \theta_{B}\right)=1-\cos \left(\theta_{A}-\theta_{B}\right)$. The same result is obtained from modeling the energy associated to a spring of zero resting length that is attached to the points $\theta_{A}$ and $\theta_{B}$ of a circle, and again by modeling the energy that is associated to a point mass that slides on a circle and that is subject to gravity; this is represented in Fig. 6.

If we adopt this definition then our total cost is $\mathcal{E}(\theta)=$ $\Sigma_{k} 1-\cos \left(\theta_{k+1}-\theta_{k}\right)$. We may minimize such "energy" or "cost" by gradient descent as follows:

$$
\begin{aligned}
\theta(k, 0) & =\theta_{0}(k) \\
\frac{\partial \theta_{k}}{\partial t} & =\lambda\left[\sin \left(\theta_{k+1} \theta_{k}\right)+\sin \left(\theta_{k-1}-\theta_{k}\right)\right] .
\end{aligned}
$$

This is the "right" way to discretize orientation diffusions.

We present the following observations.

Observation 3: The expression $\sin \left(\theta_{k+1}-\theta_{k}\right)+\sin \left(\theta_{k-1}-\right.$ $\theta_{k}$ ) may be seen as the discretization of the Laplacian for orientation-type variables $\lim _{\delta \rightarrow 0}\left(\sin \left(\theta_{k+\delta}-\theta_{k}\right)+\sin \left(\theta_{k-\delta}-\right.\right.$ $\left.\left.\theta_{k}\right) / \delta^{2}\right)=(\triangle \theta)(k)$.

Observation 4: Similarly, one may think of $\sin \left(\theta_{A}-\theta_{B}\right)$ as the "right" way to compute the finite difference on the circle. Observe that, according to this definition, the "distance" (one may think of it as a force) between zero and $\pi$ is zero. This is due to the fact that a small perturbation of the value of either angle produces only a negligible second-order change in the value of the associated energy.

Observation 5: On a square-connected bidimensional lattice the above definition of discrete laplacian generalizes to

$$
\left.\triangle \theta\right|_{(i, j)}=\sum_{n \in N(i, j)} \sin \left(\theta_{n}-\theta_{i, j}\right)
$$

where $N(i, j)$ is the set of neighbors of the lattice location $(i, j)$. This definition, using a $3 \times 3$ neighborhood structure, was used in the experiments presented in this paper. One caveat is that this 2-D definition is not rotation-invariant.

Observation 6: The energy used here is intuitive because it has a ready physical interpretation. It is by no means the only possible choice. Interesting computational behaviors may be obtained using different energy functions.

Observation 7: A contrast-invariant orientation scalespace, i.e., one that is invariant with respect to any monotonic change of image contrast $I^{\prime}=f(I)$, may be obtained if the initial conditions of the orientation diffusion are obtained by measuring the orientation of the iso-brightness curves in the image, or, more realistically, by first smoothing the image a little by mean curvature motion and then extracting the orientation of the iso-brightness curves. Orientation diffusion is obviously contrast-invariant.

\section{BIASED DIFFUSION}

At times it is useful to bias the diffusion with some data term $\theta_{d}(x, y)$. We may exploit the energy based derivation of finite differences in this case as well

$$
\begin{aligned}
\theta(x, y, 0) & =\theta_{0}(x, y) \\
\theta_{t} & =\lambda \triangle \theta+\beta \sin \left(\theta_{d}-\theta\right)
\end{aligned}
$$

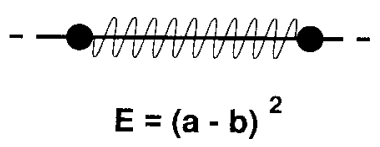

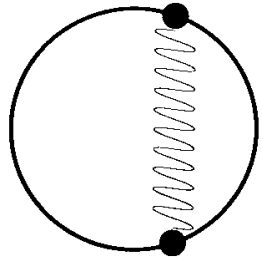

$$
E=\sin ^{2}(|a-b| / 2)
$$

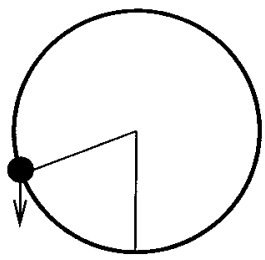

$E=1-\cos (a-b)$
Fig. 6. Energy associated to a pair of locations on the straight line (top), and to a pair of orientations (bottom). Notice that $2 \sin ^{2}\left(\theta_{A}-\theta_{B} / 2\right)=1-\cos \left(\theta_{A}-\theta_{B}\right)$, therefore both physical systems are governed by the same energy.

where $\triangle$ denotes either the Laplacian, or its discrete version as derived above.

\section{NONLINEARITY IN THE INTERACTION}

Observe the nonlinearity that is intrinsic in this diffusion scheme. While in the brightness diffusion the energy is quadratic, and therefore the flux, i.e., the amount of brightness flowing through a surface in the unit time, is proportional to the difference between neighbors, in the orientation diffusion that we just defined the flux is $\sin \left(\theta_{A}-\theta_{B}\right)$, and therefore depends nonlinearly on the difference $\theta_{A}-\theta_{B}$. This is reminiscent of the nonlinear probability density, energy and diffusion terms proposed by Geman and Geman [11], Blake and Zisserman [12], Perona and Malik [13], and others.

Notice that when $\theta_{A}-\theta_{B}=\pi / 2$ one has maximum flux, i.e., maximum interaction between neighboring orientations, while when $\theta_{A}-\theta_{B}=\pi$ the flux is zero. In this latter case the two nodes of the lattice do not interact, as if a discontinuity process [11], [12] had intervened. As pointed out earlier, the energy function that is used here is by no means the only possibility, although it may be thought of as the simplest, or the most "natural" one. More aggressive nonlinear behaviors may be obtained using, e.g., an energy that is the square of the 'natural' one.

One further observation: If the flux was not zero when neighboring lattice locations differ by $\pi$, the flux would be discontinuous there: because of symmetry it needs to change sign when the difference passes from from $\pi-\epsilon$ to $\pi+\epsilon$.

\section{UNCERTAINTY}

It is often the case that orientations may be measured with a variable level of accuracy. For example, an image may contain strongly oriented structure (edges, wood texture) and unoriented patches as well (sand texture, clouds, flat sky). As a result the data that the diffusion is "filtering" or "interpolating" has a variable degree of reliability.

For this reason, it is useful at times to incorporate in the diffusion a measure of two kinds of uncertainty: 1) the 

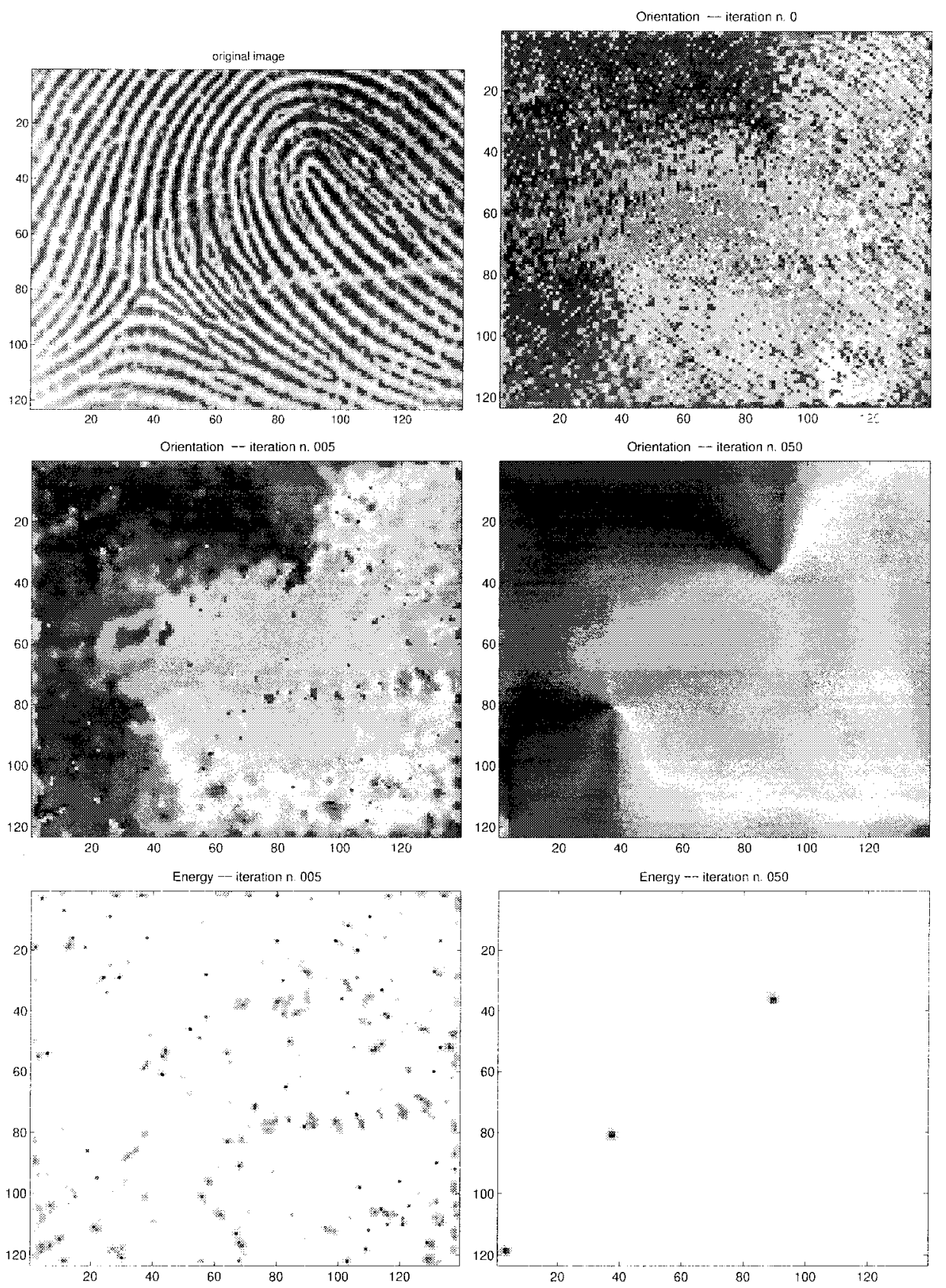

Fig. 7. Results of running orientation diffusion. The original image is shown at top left. The corresponding orientation, computed from the brightness gradient, is shown at top right. The orientation image (top-right) was used as the initial conditions of the diffusion. The second and third rows show the results of running the diffusion for five and 50 iterations (left and right columns). The second row shows the orientation and the third the energy.

uncertainty of the data in the bias term; and 2) the degree of approximation with which the diffusion has estimated local orientation.

There are a number of different approaches to this problem. We examine here some simple ideas.

\section{A. Uncertainty in the Data}

At times a model of the uncertainty with which orientation may be measured in the image is available, and this may vary throughout the image. Consider for instance the image in Fig. 1: In the white background region it is very difficult to make out any orientation, while in the fingerprint area orientation is relatively easy to measure almost everywhere. One may want to incorporate this information in the diffusion process.

One way to represent uncertainty in the data is via a probability density function $f_{\Theta_{d}}(\theta)$. The "cost" in the system composed by a deterministic orientation $\theta$ and such an uncertain orientation $\theta_{d}$ may be calculated as the expectation of the energy

$$
\begin{aligned}
\mathcal{C}\left(\theta, f_{\Theta_{d}}\right) & =\operatorname{E} \mathcal{E}\left(\theta, f_{\Theta_{d}}\right) \\
& =\int_{-\pi}^{\pi} \mathcal{E}(\theta, \phi) f_{\Theta_{d}}(\phi) d \phi .
\end{aligned}
$$



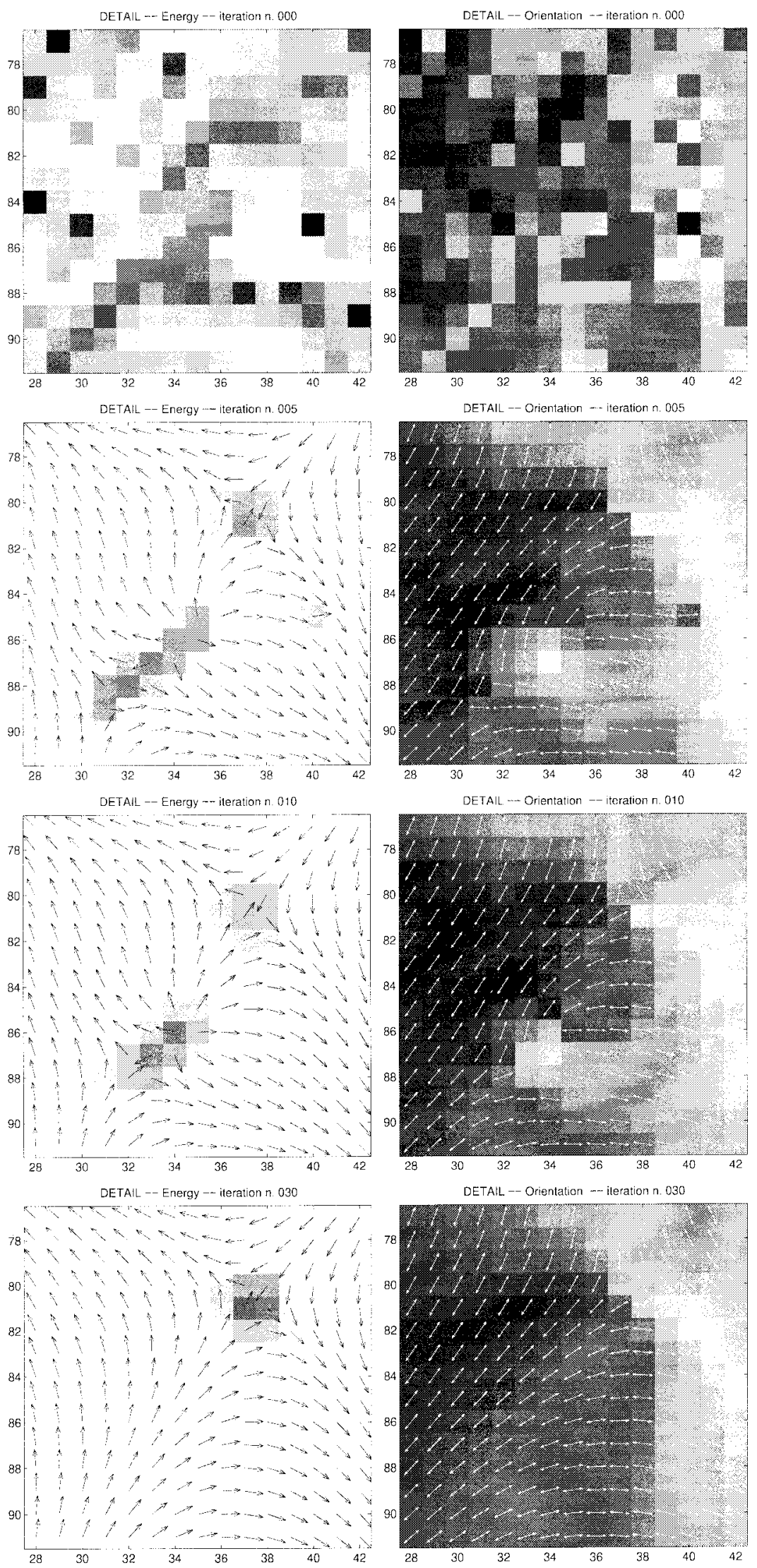

Fig. 8. Detail of the diffusion shown in Fig. 7. Zero, 5, 10, and 30 iterations are shown in rows 1-4. Orientation (left column) and energy (right column) are shown. The arrows that are superimposed on the energy and orientation images show orientation using two different conventions. Since, in fingerprints, the orientation of interest is of the line kind (see Fig. 3), such orientation may be shown via double-headed arrows that are parallel to the lines in the print (right column images), and as single-headed arrows that show the unified representation of orientation of the circle. In this case the single-headed arrows are oriented as $2 \theta$, where $\theta$ is the orientation of the underlying line. Notice the two singularities that merge and disappear briefly after iteration 10 . 

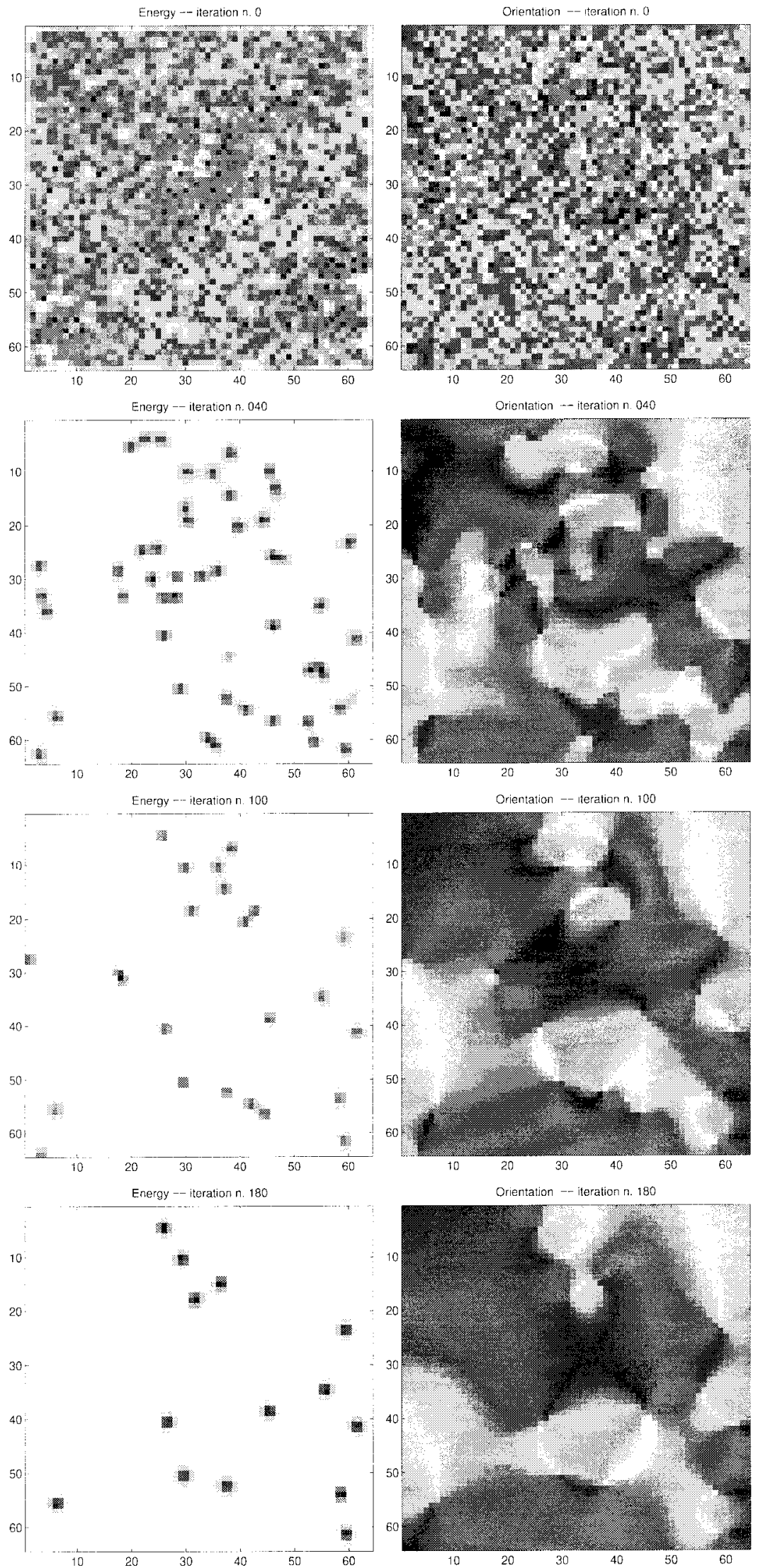

Fig. 9. The causal behavior of orientation diffusion is evident in this experiment where an image composed of random orientations was diffused. Notice that the orientation singularities are points and that they disappear, usually in pairs, as diffusion progresses. No "birth" of singularities may be observed. 

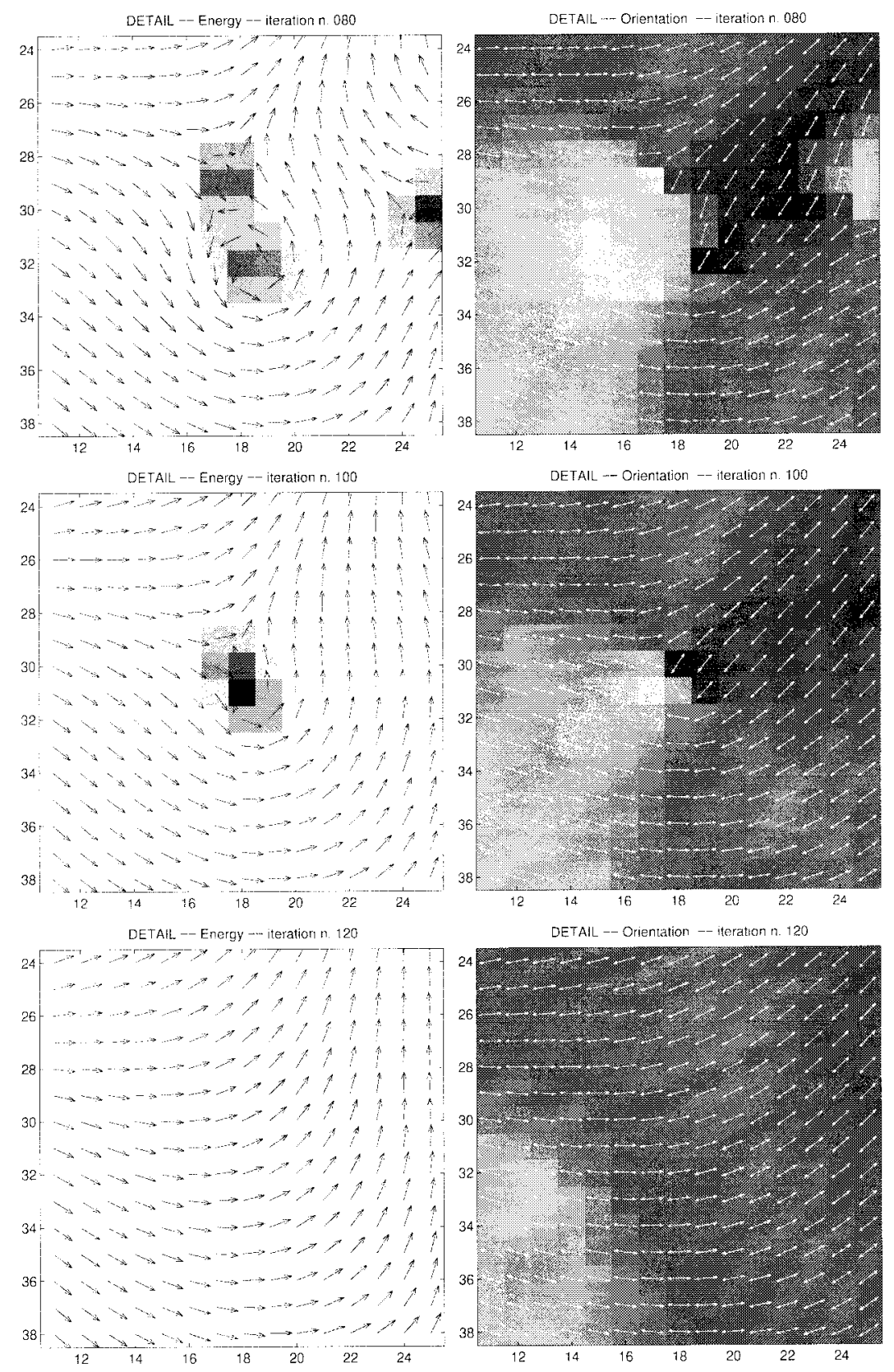

Fig. 10. Detail of the experiment of Fig. 9. The two singularities in the center of the picture merge and disappear.

It is often reasonable to assume that $\theta_{d}$ is distributed uniformly in the interval $\theta_{d} \in\left(\mu_{d}-\sigma_{d}, \mu_{d}+\sigma_{d}\right)$, i.e. $f_{\Theta_{d}}(\theta)=1 / 2 \sigma_{d}$ for $\theta$ in the interval, and zero elsewhere. In this case

$$
\begin{aligned}
\mathcal{C}\left(\theta, \theta_{d}\right) & =1-\frac{1}{2 \sigma_{d}} \int_{\mu_{d}-\sigma_{d}}^{\mu_{d}+\sigma_{d}} \cos (\phi-\theta) d \phi \\
& =1-\sin \left(\mu_{d}+\sigma_{d}-\theta\right)+\sin \left(\mu_{d}-\sigma_{d}-\theta\right) \\
& =1-\frac{\sin \sigma_{d}}{\sigma_{d}} \cos \left(\mu_{d}-\theta\right) .
\end{aligned}
$$

From this we may derive the expression for the bias term in the diffusion

$$
\begin{aligned}
\theta(x, y, 0) & =\theta_{0}(x, y) \\
\theta_{t} & =\lambda \triangle \theta+\beta \frac{\sin \sigma_{d}}{\sigma_{d}} \sin \left(\mu_{d}-\theta\right) .
\end{aligned}
$$

Observe that what we have obtained is very similar to the previous diffusion; we have just added a constant weight term $w\left(\sigma_{d}\right)=\sin \sigma_{d} / \sigma_{d}$ that accounts for the certainty of the data. As one would expect when $\sigma_{d} \rightarrow 0$ (no uncertainty) then $w\left(\sigma_{d}\right) \rightarrow 1$ and when $\sigma_{d}=\pi$ (maximal uncertainty) then $w\left(\sigma_{d}\right) \rightarrow 0$.

In the case that an uncertainty is also associated with the diffused $\theta$ then one obtains an additional multiplicative term $\sin \sigma / \sigma$.

\section{B. Uncertainty in the Value of the Diffusion}

The local energy may be useful to calculate the "reliability" $\mathcal{E}_{F}$ of the value of $\theta(x, y, t)$, and the distance $\mathcal{E}_{d}(x, y, t)$ of $\theta$ 
from the underlying data $\theta_{d}$ :

$$
\begin{aligned}
& \mathcal{E}_{F}(x, y, t)=\sum_{n \in \mathcal{N}(x, y)} 1-\cos \left(\theta_{n}(t)-\theta(x, y, t)\right) \\
& \mathcal{E}_{d}(x, y, t)=1-\frac{\sin \sigma_{d}}{\sigma_{d}} \cos \left(\mu_{d}-\theta(x, y, t)\right)
\end{aligned}
$$

where the uniform probability density described in the previous section is assumed for convenience.

The probabilistic interpretation of these energies is not clear. However, they prove to be useful, e.g., for detecting and locating the singularities in the orientation as discussed in Section VII and as shown in Fig. 7.

\section{EXPERIMENTS}

\section{A. Smoothing of Orientation}

The lattice implementation of the orientation diffusion, with the discrete Laplacian, as described by (8), was tested on the striped portion of the fingerprint image shown in Fig. 1. The intitial conditions of the diffusion, i.e., the initial orientation, were calculated from the gradient of the image brightness (orientation of the gradient plus $90^{\circ}$ ) shown in Fig. 2. The boundary conditions were adiabatic (i.e., zero-gradient).

The results of running the diffusion may be seen in Fig. 7. Notice that the diffusion has the desired effect of smoothing orientation. The two singularities of orientation that are present in the fingerprint would be difficult to detect in the original image using a local operator. They are clearly visible in the smoothed orientation image after 50 iterations (second row, second column of Fig. 7); they may be easily detected and extracted from the corresponding energy image (third row, second column of Fig. 7).

\section{B. Scale-Space and Causality}

In Fig. 8, a detail of the previous experiment is shown. The detail corresponds to a portion of the image surrounding the bottom-left orientation singularity of the fingerprint. The original orientation image (top right) is quite messy, however, after five iterations of the diffusion process (second row) most of the picture is already smooth. In the energy image (second row, left) a number of high-energy locations are visible. The rightmost one is due to a misaligned orientation which is realigned between iterations five and ten. Three more, due to "fundamental" singularities in orientation, are clearly visible. The bottom-left two singularities display an interesting and commonly observed behavior: They merge and vanish shortly after iteration ten. By iteration 30, only one singularity has remained, the one that is present in the data at a coarse scale, i.e., the delta singularity.

In the scale-space literature this behavior is called causal; singularities may disappear, but not appear, as a result of running the diffusion forward in time. When a singularity is found at a certain time, its "cause" may always be found at previous times.

In order to better explore whether the causality behavior that one observes in Fig. 8 is intrinsic to the equation, we have run a number of experiments using randomly oriented initial conditions. Violations of causality were never observed. One such experiment is shown in Fig. 9. A detail of Fig. 9 showing two singularities merging is shown in Fig. 10.

\section{DISCUSSION AND CONCLUSIONS}

It was shown that the usual definition of diffusion, such as is used on brightness data in computer vision, may be extended trivially to the case of orientations in the continuous case; however, the discrete case is particularly problematic. A common definition of orientation diffusion, obtained by embedding orientation in the plane, and then diffusing, was shown not to enjoy the semigroup property and to produce large-scale degradations of the topology of the solution.

A definition of orientation diffusion was suggested; it is based on gradient descent of an energy function inspired by the model of simple physical systems. It is defined on a lattice and it may be implemented with simple calculations on 2-cliques. Experiments conducted on the image of a fingerprint demonstrate that this diffusion de-noises orientation data effectively.

The scale-space behavior of this equation was explored experimentally. Our empirical observations may be summarized in the following three conjectures.

1) The generic singularities are points rather than lines.

2) The diffusion has the causality property, in that pointsingularities may disappear but never appear.

3) The point-singularities disappear by either merging with other singularities or by flowing off the boundary of the diffusion.

No proof of these conjectures is known to the author.

A few further questions remain open.

1) The definition of the orientation diffusion in the continuum was given for smooth solutions only. It is quite useless as such. It would be important and probably interesting to try and extend the definition to the case where solutions are allowed to have singularities, i.e., points where the orientation is discontinuous. At such points the energy function diverges, e.g., in the neighborhood of a point from which orientations radiate outwards, the energy diverges with the cube of the distance from such point. However, it is not clear that the velocity with which the solution of the diffusion evolves should be infinite; one would expect that orientations symmetrically radiating from a point would be a stable equilibrium for the equation. Unlike the case of brightness diffusions we would therefore have a number of nontrivial stable equilibria, and some such equilibria might be "dynamic" in that the corresponding "orientation flux" would be nonzero.

2) While the definition of singularities in the continuous case is clear, the term "singularity" only has an intuitive meaning in the discrete case (i.e., when the domain of definition of the orientation function is a lattice). It is unclear wether a rigorous and meaningful definition might be found.

3) Singularities come in different varieties: points from which orientations radiate outwards, points that are 
encircled by orientations, parabolae, etc. Many of these were observed by Granlund and collaborators. It would be interesting to collect a complete taxonomy of such singularities, and relate this taxonomy to the scale-space behavior of pairs of singularities. This would parallel the catastrophe theory that was developed in the case of brightness for linear scale-space [2], [14]-[16]. Work on harmonic maps and liquid crystals, e.g., [17], may shed some light on this issue.

\section{ACKNOWLEDGMENT}

The second proof was derived in collaboration with A. Salden. Conversations with S. Belongie, J. Weichert, S. Mitter, and F. Fagnani are gratefully acknowledged. Comments from the anonymous reviewers were very helpful in improving a previous version of the paper.

\section{REFERENCES}

[1] P. Perona, "Deformable kernels for early vision," IEEE Trans. Pattern Anal. Machine Intell., vol. 17, pp. 488-499, 1995.

[2] J. J. Koenderink, "The structure of images," Biol. Cybern., vol. 50, pp. $363-370,1974$

[3] A. P. Witkin, "Scale-space filtering," in Proc. Int. Joint Conf. Artificial Intelligence, Karlsruhe, Germany, 1983, pp. 1019-1021.

[4] P. Kube and P. Perona, "Scale-space properties of quadratic feature detectors," IEEE Trans. Pattern Anal. Machine Intell., vol. 18, pp. 987-999, 1996.

[5] B. M. ter Haar Romeny, Ed., Geometry-Driven Diffusion in Computer Vision. Boston, MA: Kluwer, 1994.

[6] M. Kass and A. Witkin, "Analyzing oriented patterns," Comput. Vis., Graph. Image Processing, vol. 37, pp. 362-385, 1987.

[7] A. R. Rao and B. G. Schunck, "Computing oriented texture fields," Comput. Vis., Graph., Image Processing, vol. 53, pp. 157-185, 1991.

[8] J. Bigün, G. H. Granlund, and J. Wiklund, "Multidimensional orientation estimation with applications to texture analysis and optical flow," IEEE Trans. Pattern Anal. Mach. Intell., vol. 13, no. 8, pp. 775-790, 1991.

[9] G. H. Granlund and H. Knuttson, Signal Processing for Computer Vision. Boston, MA: Kluwer, 1995

[10] J. Weickert, "Foundations and applications of nonlinear anisotropic diffusion filtering," Zeitschr. Angewandte Math. Mechan., vol. 76, pp. 283-286, 1996
[11] S. Geman and D. Geman, "Stochastic relaxation, Gibbs distributions, and the Bayesian restoration of images," IEEE Trans. Pattern Anal. Machine Intell., vol. PAMI-6, pp. 721-741, Nov. 1984.

[12] A. Blake and A. Zisserman, Visual Reconstruction. Cambridge, MA: MIT Press, 1987

[13] P. Perona and J. Malik, "Scale-space and edge detection using anisotropic diffusion," IEEE Trans. Pattern Anal. Machine Intell., vol. 12, pp. 629-639, July 1990.

[14] A. Yuille and T. Poggio, "Scaling theorems for zero crossings," IEEE Trans. Pattern Anal. Machine Intell., vol. 8, pp. 15-26, Jan. 1986.

[15] J. Babaud, A. Witkin, Baudin, and R. Duda, "Uniqueness of the Gaussian kernel for scale-space filtering," IEEE Trans. Pattern Anal. Machine Intell., vol. PAMI-8, pp. 26-33, Jan. 1986.

[16] J. J. Clark, "Authenticating edges produced by zero-crossing algorithms," IEEE Trans. Pattern Anal. Machine Intell., vol. 11, pp. 55-73, Jan. 1989.

[17] J.-M. Coron, H. Brezis, and E. Lieb, "Harmonic maps with defects," Commun. Math. Phys., 1986.

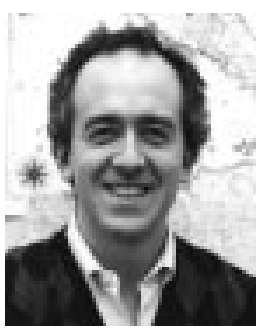

Pietro Perona received the D.Eng degree in electrical engineering from the University of Padova, Italy, in 1985 , and the Ph.D. degree in electrical engineering from the University of California, Berkeley, in 1990

$\mathrm{He}$ is currently a Professor of electrical engineering and computation and neural systems at the California Institute of Technology (Caltech), Pasadena. He is also an Adjunct Associate Professor with the University of Padova, Italy. He is also the Deputy Director of the National Science Foundation Engineering Research Center on Neuromorphic Systems Engineering, Caltech, and the U.S. coordinator for the NSF-ESPRIT-funded study group on geometry driven diffusion. His research interests include both human and machine vision. His current activity is focused on the study of vision as a sensor for control and estimation applications ("dynamic vision"), the use of vision as a sensor for human-machine interfaces, in particular for tracking the motion of the human body, visual object recognition, and the perception of shape from shading. He has also worked on the use of diffusive PDE's for image processing (anisotropic diffusion) and filtering techniques for early vision and modeling of human vision.

Dr. Perona received the National Young Investigator Award in 1994. He serves as a referee in numerous scientific journals, and as a member of the program committees for major vision conferences. 